\title{
Solid Coverings of the Euclidean Plane with Incongruent Circles*
}

\author{
A. Florian ${ }^{1}$ and A. Heppes ${ }^{2}$ \\ ${ }^{1}$ Institut für Mathematik, Universität Salzburg, \\ Hellbrunnerstrasse 34, A-5020 Salzburg, Austria \\ August.Florian@sbg.ac.at \\ ${ }^{2}$ Vércse u. 24/A, H-1124 Budapest, Hungary \\ h9202hep@ella.hu
}

Communicated by Imre Bárány

\begin{abstract}
In this paper we consider coverings of the plane by circles of two different sizes. We establish a sufficient condition for such a covering to be solid in the sense of L. Fejes Tóth [6]. As an application of this general theorem we prove that there exist infinitely many solid coverings of this kind.
\end{abstract}

\section{Introduction}

A set of open (closed) circles is said to form a packing (covering) of the Euclidean plane if each point of the plane belongs to at most (at least) one circle of the set. Packings and coverings of the plane with incongruent circles are the subject of several papers (see, e.g., [5] and [9]-[11]) and of the comprehensive monograph [7]. One of the results is that the density of any packing (covering) with circles of not too different radii is not greater (not less) than the maximum (minimum) density of packings (coverings) with congruent circles. This is a nonpublished result of K. Böröczky (for ref. see p. 194 of [7]). It was independently established and published by Blind [2] and is contained in a more general theorem of G. Fejes Tóth [3]. The upper and lower bounds for the packing and covering densities of the arrangements consisting of circles with radii selected from a given bounded interval are in some cases very close to each other. In this connection, many interesting packings and coverings with circles of different sizes have been suggested (see [16]). However, none of them—with the exception of Böröczky's and Blind's results—

\footnotetext{
* The first author wishes to thank the Austrian and Hungarian Academies of Science for supporting a visit to Budapest in May 1997.
} 


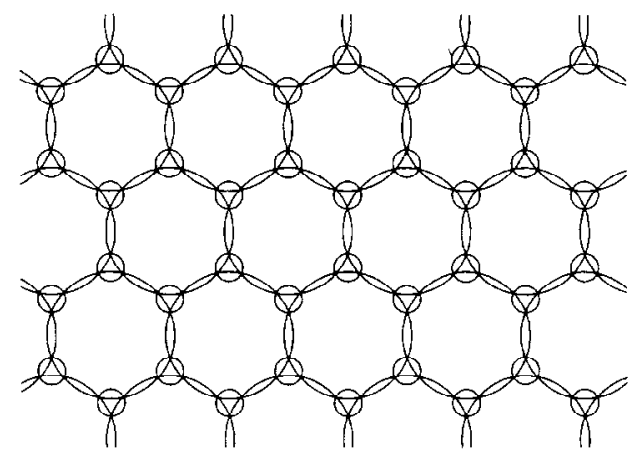

Fig. 1

could be proved to be extremal among all packings or coverings by circles with radii selected from a given interval.

On the other hand, Florian [12] considered coverings involving two types of circles of radius 1 and $p<1$, respectively. He found an arrangement of minimal density for $p=p_{1}=0.2483 \ldots$, and for $p=p_{2}=0.5023 \ldots$ (see Figs. 1 and 2 ). Recently, it has been shown that any covering of the plane with two kinds of circles has a density $\geq 1.01895 \ldots$. [5]. There are coverings of the plane with two kinds of circles whose densities are arbitrarily close to this constant.

The investigation gained fresh impetus when L. Fejes Tóth introduced the notion of solidity. A packing (covering) of disks is said to be solid if no finite number of its members can be rearranged so as to form, together with the rest of the members, a packing (covering) not congruent to the original one. Solid sets of circles were investigated by several authors [6], [4], [1], [8], [15]. Some promising configurations, e.g., the set of incircles of the Archimedean tiling $(4,8,8)$, resisted for a long time all attempts to prove them to be solid. Recently, Heppes [14] succeeded in proving the solidity of a number of packings consisting of two or three kinds of circles, including the case $(4,8,8)$.

In this paper we combine the methods employed by the two authors to investigate the solidity of coverings. The coverings considered here consist of circles of two different

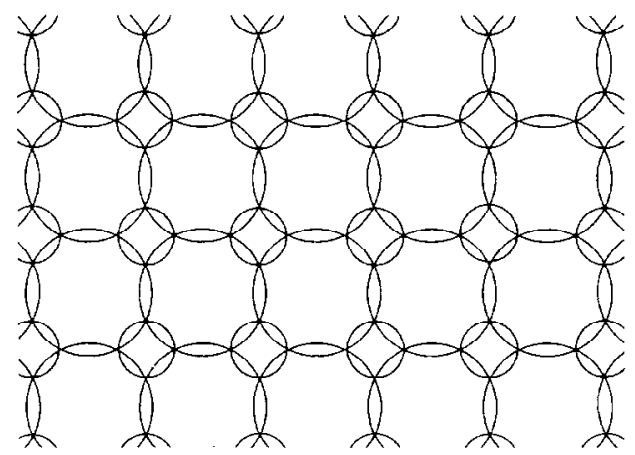

Fig. 2 
sizes, e.g., of radius 1 and $p<1$. A sufficient condition for the solidity of such coverings is established. As an application of this general theorem we prove the existence of infinitely many solid coverings of this kind. More precisely, there exist two intervals for $p$ (i.e., the radius of the smaller circle), one containing the previously mentioned value $p_{1}=0.2483 \ldots$, and another containing $p_{2}=0.5023 \ldots$, such that for each value inside these intervals a solid covering can be found.

\section{A General Theorem}

In order to state our main result, we introduce some notions referring to coverings of the plane with circles.

Throughout this paper it is assumed that the covering is irreducible, i.e., none of the circles is superfluous (to each circle there exists a point in the plane that is covered only by the circle in question). A covering containing superfluous circles is clearly nonsolid. We consider an irreducible covering with circles of radii from a given interval $[a, b]$, where $a>0$, and make the additional assumption that each circle of the plane contains only a finite number of their centers. Again, a covering that fails to satisfy this condition is nonsolid, as there would be a bounded region containing infinitely many circles of the covering, while a finite number of them could be arranged so as to cover this region.

The covering generates a Delaunay triangulation of the plane, as described in [9]. We obtain an edge-to-edge tiling, whose faces are triangles and whose vertices are the centers of the circles. The three circles centered at the vertices of the same triangle have a point in common (this implies that their union covers the triangle). We mark each tile by assigning to each vertex the radius of the circle of the covering centered at this vertex. In this sense we say that a covering with circles generates a marked tiling (see [13]).

Let $t$ be a triangle spanned by the centers $O_{1}, O_{2}, O_{3}$ of three circles with radii $r_{1}, r_{2}, r_{3}$, respectively. We assign a positive weight $w\left(r_{i}\right)$ to the circle of radius $r_{i}$ and define the function

$$
\delta=\frac{\sum_{i=1}^{3} r_{i}^{2} \alpha_{i} w\left(r_{i}\right)}{2 \Delta},
$$

where $\alpha_{i}$ is the angle of $t$ at $O_{i}(i=1,2,3)$ and $\Delta$ denotes the area of $t$. It is reasonable to call $\delta$ the "weighted density" of the three circles in $t$, or, in short, the density in $t$ (see [14]). Note that we do not require the sectors of the circles to lie completely in $t$.

Let $R$ be a set of radii, and let a positive weight be assigned to each element of $R$. We denote the set of these assigned weights by $W$. A circle whose radius belongs to $R$ is said to be admissible. For a given set $R$ and an assigned set $W$, we consider all triangles whose vertices are centers of admissible circles having a point in common. The triangles of minimal density (in the above sense) are called light triangles defined by the sets $R$ and $W$. Observe that there can be several types of light triangles, as the solution of this extremum problem is possibly not unique (see [14]). If $R$ is a finite set (as in the theorem below), then light triangles do exist.

A marked tiling consisting of light triangles is said to be solid if, for any replacement of a finite number of its tiles by a finite number of light triangles which leads to a properly marked tiling, the set of the marked vertices is the same. In our case, the term "properly 
marked" means that the circular sectors associated with the angles of the tiles fit together to form complete circles (or, in other words, all corners of tiles meeting at a point bear identical marks).

Now we can state the following

Theorem 1. Let $\mathcal{C}$ be an irreducible covering consisting of circles with radii $r_{1}>0$ and $r_{2}>0$. Then $\mathcal{C}$ is solid if the following two conditions are satisfied:

(i) Positive weights $w_{1}$ and $w_{2}$ can be assigned to the circles of radius $r_{1}$ and $r_{2}$, respectively, in such a way that the marked tiling generated by $\mathcal{C}$ consists of light triangles defined by the sets $\left\{r_{1}, r_{2}\right\}$ and $\left\{w_{1}, w_{2}\right\}$.

(ii) The marked tiling generated by $\mathcal{C}$ is solid.

\section{Preliminaries}

In this section we establish some properties of light triangles in the case where there are only two types of admissible circles.

Let $C_{1}, C_{2}, C_{3}$ be three circles with radii $r_{1}, r_{2}, r_{3}$ centered at the vertices of the triangle $t=O_{1} O_{2} O_{3}$, and we assume that the circles have a point $P$ in common. If $P$ is outside $t$, then the point of $t$ nearest to $P$ belongs also to $C_{1} \cap C_{2} \cap C_{3}$. Thus we can assume that $P \in t$. If the intersection of the three circles has an interior point, then $t$ can be enlarged, and thus the weighted density can be decreased while the circles continue to have a point in common. Therefore, in a light triangle the circles have a single point in common, and this point lies in the interior or on the boundary of $t$. This presupposition is assumed in the following lemma.

Symmetry Lemma. Consider three circles with radii $r_{1}, r_{2}=r_{1}, r_{3}$ centered at the vertices of the triangle $t=\mathrm{O}_{1} \mathrm{O}_{2} \mathrm{O}_{3}$. Assume that the circles have a single point $P$ in common. Let $r$ and $w$ be positive numbers, and let $\{1, r\}$ and $\{1, w\}$ be the set of admissible radii and assigned weights, respectively. If the triangle $t$ is not isosceles itself, with apex at $\mathrm{O}_{3}$ and $P$ lying on the boundaries of all three circles, then there exists an isosceles triangle $t^{\prime}=O_{1}^{\prime} O_{2}^{\prime} O_{3}^{\prime}$, with apex at $O_{3}^{\prime}$, such that the circles with radii $r_{1}, r_{2}, r_{3}$, centered at $O_{1}^{\prime}, O_{2}^{\prime}, O_{3}^{\prime}$, have a single point in common, which lies on the boundaries of all three circles, and the weighted density in $t^{\prime}$ is smaller than that in $t$.

Proof. Let $C_{i}$ be the circle with center $O_{i}(i=1,2,3)$. If $P$ is a single point in common, then it lies on the boundaries of at least two of the circles. If $P$ lies in the interior of the third circle, then-keeping $\alpha_{3}$, the angle at $O_{3}$, fixed-this "loose" circle can be moved (thereby increasing the area of $t$ ) until either $P$ becomes a boundary point of the third circle as well or the required symmetric position is reached. If the loose circle is $C_{1}$, then the center of $C_{1}$ will be moved away from $O_{3}$ on the half-line $O_{3} O_{1}$ until $P$ reaches the boundary of $C_{1}$, and similarly for the loose circle $C_{2}$. If the loose circle is $\mathrm{C}_{3}$, then $\mathrm{O}_{3}$ will be moved on the circumcircle of $t$ away from the base $O_{1} O_{2}$ until either $P$ becomes a boundary point of $\mathrm{C}_{3}$ or the triangle becomes isosceles with apex at $\mathrm{O}_{3}$. Since the weighted angle sum is constant in either case, the weighted density decreases. 
If $C_{3}$ is still loose, then a further operation is applied: the isosceles triangle is replaced by another one of height $r_{3}$ and unchanged base of length $2 r_{1}$. If the base angles are $\alpha_{1}$, the weighted density is

$$
\frac{2 r_{1}^{2} \alpha_{1} w\left(r_{1}\right)+r_{3}^{2} \alpha_{3} w\left(r_{3}\right)}{2 r_{1}^{2} \tan \alpha_{1}}=\frac{\alpha_{1}}{\tan \alpha_{1}} w\left(r_{1}\right)+\frac{\pi-2 \alpha_{1}}{\tan \alpha_{1}} \frac{r_{3}^{2} w\left(r_{3}\right)}{2 r_{1}^{2}}
$$

which is the sum of two strictly decreasing functions of $\alpha_{1}$. Hence the weighted density strictly decreases again.

For the rest of the proof we can assume that $P$ is a single point common to and lying on the boundaries of all three circles.

There are four possible cases, each of which corresponds to a choice of admissible radii:

- Case $(1,1,1): r_{1}=1, r_{2}=1$, and $r_{3}=1$.

- Case $(1,1, r): r_{1}=1, r_{2}=1$, and $r_{3}=r$.

- Case $(r, r, 1): r_{1}=r, r_{2}=r$, and $r_{3}=1$.

- Case $(r, r, r): r_{1}=r, r_{2}=r$, and $r_{3}=r$.

In Case $(1,1,1)$ as well as in Case $(r, r, r)$ the three circles are congruent and the weighted angle sum is constant. Therefore, the weighted density attains its minimum if and only if $\mathrm{O}_{1} \mathrm{O}_{2} \mathrm{O}_{3}$ is an equilateral triangle.

The remaining two cases, $(1,1, r)$ and $(r, r, 1)$, are considered separately. One can assume that $r<1$, since for $r>1$ each of these cases is equivalent to the other for $r<1$.

Case $(1,1, r)$. Let $O_{1}^{\prime}=O_{1}, O_{2}^{\prime}=O_{2}$, and $O_{3}^{\prime}=Q$, where $Q$ denotes that point of the circumcircle of $\mathrm{O}_{1} \mathrm{O}_{2} \mathrm{O}_{3}$ which is farthest from $\mathrm{O}_{1} \mathrm{O}_{2}$ (Fig. 3). This transformation increases the area of $t$; it also leaves $\alpha_{3}$ (and thus the weighted angle sum) unchanged and the point $P$ covered (because $r<1$ ). With another appropriate enlargement of the triangle, the circles will have a single point in common.

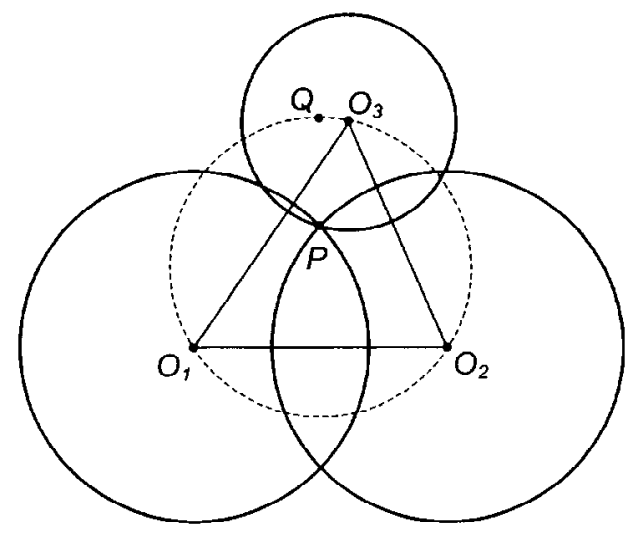

Fig. 3 
Case $(r, r, 1)$. The weighted density of the three circles is

$$
\delta=\frac{\left(\alpha_{1}+\alpha_{2}\right) w r^{2}+\alpha_{3}}{2 \Delta}
$$

If $P$ is the common boundary point of the circles, we write $\varphi_{1}, \varphi_{2}$, and $\varphi_{3}$ for the angles $\mathrm{O}_{2} \mathrm{PO}_{3}, \mathrm{O}_{3} \mathrm{PO}_{1}$, and $\mathrm{O}_{1} \mathrm{PO}_{2}$, respectively. Then

$$
\delta=\frac{\pi w r^{2}+\left(1-w r^{2}\right) \alpha_{3}}{r\left(\sin \varphi_{1}+\sin \varphi_{2}\right)+r^{2} \sin \varphi_{3}},
$$

where $0<\varphi_{i} \leq \pi(i=1,2,3)$ and $\varphi_{1}+\varphi_{2}+\varphi_{3}=2 \pi$. For $\alpha_{3}$ we find

$$
\alpha_{3}=\operatorname{arccot} \frac{1-r \cos \varphi_{1}}{r \sin \varphi_{1}}+\operatorname{arccot} \frac{1-r \cos \varphi_{2}}{r \sin \varphi_{2}},
$$

where $0<\operatorname{arccot} x<\pi$.

In the following, $\varphi_{3}$ is kept fixed, $\varphi_{1}$ is variable, and $\varphi_{2}=2 \pi-\varphi_{1}-\varphi_{3}$. Making use of

$$
\frac{d}{d \varphi} \operatorname{arccot} \frac{1-r \cos \varphi}{r \sin \varphi}=-\frac{r^{2}-r \cos \varphi}{1-2 r \cos \varphi+r^{2}}
$$

we find

$$
\frac{d \alpha_{3}}{d \varphi_{1}}=\frac{r\left(1-r^{2}\right)\left(\cos \varphi_{1}-\cos \varphi_{2}\right)}{\left(1-2 r \cos \varphi_{1}+r^{2}\right)\left(1-2 r \cos \varphi_{2}+r^{2}\right)} .
$$

To prove the lemma, we shall show that $\delta^{\prime}\left(\varphi_{1}\right)<0$ for $\pi-\varphi_{3}<\varphi_{1}<\varphi_{2}$, where $\delta\left(\varphi_{1}\right)$ is given by (3). Straightforward calculation yields

$$
\left[r\left(\sin \varphi_{1}+\sin \varphi_{2}\right)+r^{2} \sin \varphi_{3}\right]^{2} \delta^{\prime}\left(\varphi_{1}\right)=r\left(\cos \varphi_{1}-\cos \varphi_{2}\right) f\left(\varphi_{1}\right)
$$

with

$$
\begin{aligned}
f\left(\varphi_{1}\right)= & {\left[r\left(\sin \varphi_{1}+\sin \varphi_{2}\right)+r^{2} \sin \varphi_{3}\right] \frac{\left(1-r^{2}\right)\left(1-w r^{2}\right)}{\left(1-2 r \cos \varphi_{1}+r^{2}\right)\left(1-2 r \cos \varphi_{2}+r^{2}\right)} } \\
& -\left[\pi w r^{2}+\left(1-w r^{2}\right) \alpha_{3}\right] .
\end{aligned}
$$

If $1-w r^{2} \leq 0$, then $f\left(\varphi_{1}\right)<0$ and $\delta^{\prime}\left(\varphi_{1}\right)<0$ for $\varphi_{1}<\varphi_{2}$. Henceforth we can assume that

$$
1-w r^{2}>0
$$

From (4) it follows that

$$
\begin{aligned}
f^{\prime}\left(\varphi_{1}\right)= & {\left[r\left(\sin \varphi_{1}+\sin \varphi_{2}\right)+r^{2} \sin \varphi_{3}\right]\left(1-r^{2}\right)\left(1-w r^{2}\right) } \\
& \times \frac{4 r \sin \left(\left(\varphi_{1}-\varphi_{2}\right) / 2\right)\left[\left(1+r^{2}\right) \cos \left(\varphi_{3} / 2\right)+2 r \cos \left(\left(\varphi_{1}-\varphi_{2}\right) / 2\right)\right]}{\left(1-2 r \cos \varphi_{1}+r^{2}\right)^{2}\left(1-2 r \cos \varphi_{2}+r^{2}\right)^{2}} .
\end{aligned}
$$


Since both of the terms in square brackets are positive, we conclude that $f^{\prime}\left(\varphi_{1}\right)<$ 0 for $\varphi_{1}<\varphi_{2}$ and that $f\left(\varphi_{1}\right)$ attains its maximum if $\varphi_{1}$ is minimal, i.e., if $\varphi_{1}=\pi-\varphi_{3}$. Hence

$$
\begin{aligned}
f\left(\varphi_{1}\right) \leq f\left(\pi-\varphi_{3}\right)= & \frac{r(1-r)\left(1-w r^{2}\right) \sin \varphi_{3}}{1+2 r \cos \varphi_{3}+r^{2}} \\
& -\left[\pi w r^{2}+\left(1-w r^{2}\right) \operatorname{arccot} \frac{1+r \cos \varphi_{3}}{r \sin \varphi_{3}}\right] .
\end{aligned}
$$

Denoting $f\left(\pi-\varphi_{3}\right)=g\left(\varphi_{3}\right)$ we see that

$$
\lim _{\varphi_{3} \rightarrow 0} g\left(\varphi_{3}\right)=\lim _{\varphi_{3} \rightarrow \pi} g\left(\varphi_{3}\right)=-\pi w r^{2}<0 .
$$

From

$$
g^{\prime}\left(\varphi_{3}\right)=\frac{r^{2}\left(1-w r^{2}\right)\left(1+\cos \varphi_{3}\right)\left(1-2 r-r^{2}-2 \cos \varphi_{3}\right)}{\left(1+2 r \cos \varphi_{3}+r^{2}\right)^{2}}
$$

we gather that $g\left(\varphi_{3}\right)$ attains its maximum in $\varphi_{3}=0$ and $\varphi_{3}=\pi$. Combining this with (5), we come to the conclusion that $g\left(\varphi_{3}\right)<0$ for $0 \leq \varphi_{3} \leq \pi$ and that $f\left(\varphi_{1}\right)<0$. This implies that $\delta^{\prime}\left(\varphi_{1}\right)<0$ for $\pi-\varphi_{3}<\varphi_{1}<\varphi_{2}$, as required.

The following lemma refers to symmetric positions of two unit circles and a third circle with arbitrary radius. A real function $f$ is said to be quasi-convex on an interval $I$ if

$$
f(\lambda x+(1-\lambda) y) \leq \max \{f(x), f(y)\}
$$

for all $x, y \in I$ and $0<\lambda<1$. Strict quasi-convexity is defined by requiring strict inequality when $x \neq y$.

Quasi-Convexity Lemma. Let $t=\mathrm{O}_{1} \mathrm{O}_{2} \mathrm{O}_{3}$ be an isosceles triangle with area $\Delta$, apex $O_{3}$, and angles $\alpha_{2}=\alpha_{1}$ and $\alpha_{3}$. We assume that the circles centered at $O_{1}, O_{2}$, and $O_{3}$ with radii 1,1 , and $r(r>0)$ have one single point $P \in t$ in common which is a boundary point of all three circles. Let $x$ be the distance of $P$ from the segment $\mathrm{O}_{1} \mathrm{O}_{2}$ (Fig. 4). We use $\left\{w_{1}, w_{3}\right\}$ to denote a set of positive weights. For any $r>0$, the weighted density in $t$

$$
\delta(x, r)=\frac{2 \alpha_{1} w_{1}+\alpha_{3} w_{3} r^{2}}{2 \Delta}
$$

is strictly quasi-convex in $0 \leq x<1$ and attains its minimum at exactly one point $0<x<1$.

Proof. The proof is similar to that of Lemma 1 in [11]. From

$$
\delta(x, r)=\frac{\pi w_{1}+2\left(w_{3} r^{2}-w_{1}\right) \arctan (y /(x+r))}{2 y(x+r)},
$$




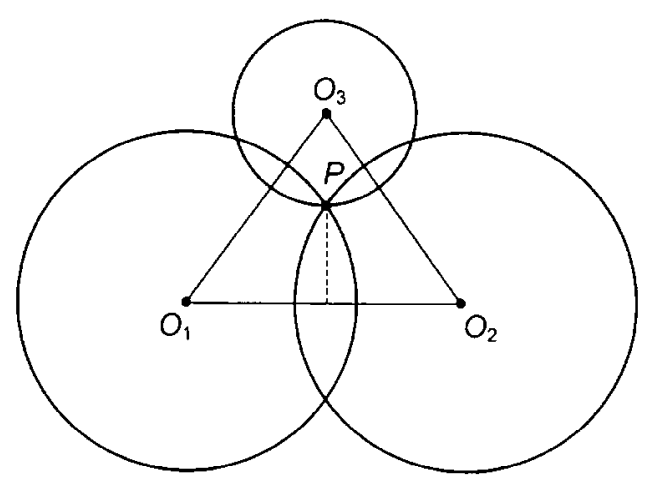

Fig. 4

where $y=\sqrt{1-x^{2}}$, it follows that

$$
\begin{aligned}
2 y^{2}(x+r)^{2} & \frac{\partial \delta}{\partial x} \\
= & y(x+r)\left[2\left(w_{3} r^{2}-w_{1}\right) \frac{(x+r)^{2}}{y^{2}+(x+r)^{2}} \cdot \frac{(x+r)(-x / y)-y}{(x+r)^{2}}\right] \\
& -\left[\pi w_{1}+2\left(w_{3} r^{2}-w_{1}\right) \arctan \frac{y}{x+r}\right] \cdot\left[-\frac{x}{y}(x+r)+y\right] \\
= & -2\left(w_{3} r^{2}-w_{1}\right)(x+r) \frac{1+r x}{y^{2}+(x+r)^{2}} \\
& +\frac{2 x^{2}+r x-1}{y}\left[\pi w_{1}+2\left(w_{3} r^{2}-w_{1}\right) \arctan \frac{y}{x+r}\right] .
\end{aligned}
$$

Note that

$$
\pi w_{1}+2\left(w_{3} r^{2}-w_{1}\right) \arctan \frac{y}{x+r}>0
$$

for all $0<x<1$. Note also that $2 x^{2}+r x-1$ has only one root $x_{0}$ in $(0,1)$ and that $2 x^{2}+r x-1<0$ or $>0$, depending on whether $0 \leq x<x_{0}$ or $x_{0}<x<1$.

We distinguish three cases:

Case 1: $w_{3} r^{2}-w_{1}=0$. In this case $\partial \delta / \partial x$ and $2 x^{2}+r x-1$ have the same sign, so that $\delta$ is strictly quasi-convex and attains its minimum at $x=x_{0}$.

Case 2: $w_{3} r^{2}-w_{1}<0$. If $x_{0} \leq x<1$, then $\partial \delta / \partial x>0$. Now let $0<x<x_{0}$. Then we have

$$
2 y^{3}(x+r)^{2} \frac{\partial \delta}{\partial x}=2\left(w_{3} r^{2}-w_{1}\right)\left(2 x^{2}+r x-1\right) f(x, r)
$$

where

$$
f(x, r)=\frac{(x+r)(1+r x) y}{\left(1-r x-2 x^{2}\right)\left[y^{2}+(x+r)^{2}\right]}+\arctan \frac{y}{x+r}+\frac{\pi}{2} \frac{w_{1}}{w_{3} r^{2}-w_{1}} .
$$


Straightforward calculation then yields

$$
\begin{aligned}
& \left(1-r x-2 x^{2}\right)^{2}\left[y^{2}+(x+r)^{2}\right]^{2} \frac{\partial f}{\partial x} \\
& =(x+r) y\left(2 r^{3}+4 x+8 r^{2} x+14 r x^{2}+4 r^{3} x^{2}+8 r^{2} x^{3}\right),
\end{aligned}
$$

which shows that

$$
\frac{\partial f}{\partial x}>0
$$

for $x \in(0,1), x \neq x_{0}$. From

$$
f(0, r)=\frac{r}{1+r^{2}}+\arctan \frac{1}{r}+\frac{\pi}{2} \frac{w_{1}}{w_{3} r^{2}-w_{1}}
$$

we see that

$$
\lim _{r \rightarrow 0} f(0, r)=0
$$

and

$$
\frac{d}{d r} f(0, r)=-\frac{2 r^{2}}{\left(1+r^{2}\right)^{2}}-\pi \frac{w_{1} w_{3} r}{\left(w_{3} r^{2}-w_{1}\right)^{2}}<0,
$$

so that $f(0, r)<0$ for $r>0$. Furthermore, since $f\left(x_{0}-0, r\right)=+\infty$, the inequality $\partial f / \partial x>0$ implies that $f(x, r)$ and $\partial \delta / \partial x$ are negative in $0<x<x_{1}$, and positive in $x_{1}<x<x_{0}$, for some $x_{1} \in\left(0, x_{0}\right)$. Thus $\partial \delta / \partial x<0$ in $0<x<x_{1}$ and $>0$ in $x_{1}<x<1$, which proves the assertion.

Case 3: $w_{3} r^{2}-w_{1}>0$. If $0<x \leq x_{0}$, then $2 x^{2}+r x-1 \leq 0$ and $\partial \delta / \partial x<0$. Let now $x_{0}<x<1$. In view of $\partial f / \partial x>0, f(1, r)=(\pi / 2)\left(w_{1} /\left(w_{3} r^{2}-w_{1}\right)\right)>0$, and $f\left(x_{0}+0, r\right)=-\infty$, we find that $f(x, r)<0$ if $x_{0}<x<x_{2}$, and $f(x, r)>0$ if $x_{2}<x \leq 1$, for some $x_{2} \in\left(x_{0}, 1\right)$.

Hence, $\partial \delta / \partial x<0$ if $0<x<x_{2}$, and $\partial \delta / \partial x>0$ if $x_{2}<x<1$, as required.

This completes the proof of the Quasi-Convexity Lemma.

\section{Proof of Theorem 1}

Let $\mathcal{C}$ be an irreducible covering of the plane with circles satisfying condition (i) of Theorem 1 . Let $\mathcal{S}$ be an arbitrary, finite, and nonempty subset of $\mathcal{C}$. We remove $\mathcal{S}$ from $\mathcal{C}$ and denote by $H$ the hole, i.e., the open bounded set generated by the removal of $\mathcal{S}$.

We proceed by stating an extremum problem for weighted areas.

Let $\mathcal{S}^{\prime \prime}$ be a finite set of admissible circles (of radius $r_{1}$ or $r_{2}$ ) satisfying the following conditions:

(a) The set $\mathcal{S}^{\prime \prime}$ covers the hole $H$ (such that $\mathcal{S}^{\prime \prime}$ together with $\mathcal{C} \backslash \mathcal{S}$ forms a covering of the plane), and

(b) the total weighted area of the circles of $\mathcal{S}^{\prime \prime}$ is as small as possible. 
Note that neither the number nor the selection of the members of $\mathcal{S}^{\prime \prime}$ is restricted, so that $\mathcal{S}^{\prime \prime}$ need not be a rearrangement of the circles of $\mathcal{S}$. Such an $\mathcal{S}^{\prime \prime}$ does indeed exist, since there is a positive lower bound to the weighted areas of admissible circles.

By conditions (a) and (b), each circle of $\mathcal{S}^{\prime \prime}$ must cover a part of the hole $H$ and is therefore lying in the outer parallel domain of $H$ of distance $\max \left\{2 r_{i}\right\}$. In the covering formed by $\mathcal{S}^{\prime \prime}$ together with $\mathcal{C} \backslash \mathcal{S}$, no circle of $\mathcal{S}^{\prime \prime}$ is redundant, whereas some circles of $\mathcal{C} \backslash \mathcal{S}$ might become superfluous. We denote the set of redundant circles by $\mathcal{R}$. As $\mathcal{C}$ is irreducible, every member of $\mathcal{R}$ intersects some circle of $\mathcal{S}^{\prime \prime}$. Thus all circles of $\mathcal{S}^{\prime \prime}$ and $\mathcal{R}$ are contained in the interior of the outer parallel domain of $H$ of distance $\max \left\{4 r_{i}\right\}$, which we denote by $H_{P}$. Observe that (for the given fixed $\mathcal{S}$ ) the domain $H_{P}$ does not depend on a special set $\mathcal{S}^{\prime \prime}$ satisfying (a) and (b). By eliminating the elements of $\mathcal{R}$ we obtain an irreducible covering $\mathcal{C}^{\prime \prime}$ consisting of the circles of $\mathcal{S}^{\prime \prime}$ and a subset of the circles of $\mathcal{C} \backslash \mathcal{S}$.

From the very nature of the triangular decomposition associated with an irreducible covering it follows that the tiling depends on the covering only locally, i.e., if the circles of the covering are replaced by other ones so that the modification is limited to a certain bounded domain $D_{1}$, then there exists another bounded domain $D_{2} \supset D_{1}$, depending only on $D_{1}$, such that outside $D_{2}$ the Delaunay tiling need not be changed. Consequently, in our case there exists a bounded domain $U$, the union of a finite subset of the triangles of the original decomposition generated by $\mathcal{C}$, which not only covers the domain $H_{P}$ (for the given fixed $\mathcal{S}$ ), but has the additional property that the marked triangles of the new covering $\mathcal{C}^{\prime \prime}$ and those of $\mathcal{C}$ can be assumed to be identical outside $U$.

We now consider the triangles of the original tiling that make up $U$. As all triangles are light, the sum of the weighted areas of the sectors of circles from $\mathcal{C}$ associated with them is equal to $\delta^{*} \cdot a(U)$, where $\delta^{*}$ denotes the density in a light triangle, and $a(U)$ denotes the area of $U$. Since $\mathcal{S}$ is covered by $U$, one set of the sectors fit together to form the complete circles of $\mathcal{S}$, while the other set belongs to circles of $\mathcal{C} \backslash \mathcal{S}$.

Now let $\mathcal{S}$ be replaced by $\mathcal{S}^{\prime \prime}$, and let $\sigma$ be the sum of the weighted areas of the circles of $\mathcal{S}^{\prime \prime}$ and of the sectors of $\mathcal{C} \backslash \mathcal{S}$ mentioned before. In view of the minimum property of $\mathcal{S}^{\prime \prime}$ we have

$$
\sigma \leq \delta^{*} \cdot a(U)
$$

By (a), $\mathcal{S}^{\prime \prime}$ together with $\mathcal{C} \backslash \mathcal{S}$ forms a covering of the plane. By eliminating the set $\mathcal{R}$ of unnecessary circles we obtain the irreducible covering $\mathcal{C}^{\prime \prime}$. We denote the sum of the weighted areas of the circles of $\mathcal{S}^{\prime \prime}$ and of the remaining sectors of $\mathcal{C} \backslash \mathcal{S}$ by $\sigma^{\prime} \leq \sigma$. Since $\mathcal{R}$ is contained in $U$, the difference $\sigma-\sigma^{\prime}$ gives the total weighted area of the circles of $\mathcal{R}$.

The covering $\mathcal{C}^{\prime \prime}$ generates a new marked tiling consisting of triangles. According to the choice of $U$, the marked triangles outside $U$ are identical to those of $\mathcal{C}$. In the new decomposition of the plane, $U$ itself is the union of a finite set of new triangles, say $T_{1}, \ldots, T_{n}$. Every $T_{i}$ is associated with three sectors of circles from $\mathcal{C}^{\prime \prime}$, and $T_{1}, \ldots, T_{n}$ are joining properly. Because $\mathcal{S}^{\prime \prime}$ is covered by $U$, one set of the sectors fits together to constitute the complete circles of $\mathcal{S}^{\prime \prime}$, and the other set belongs to circles of $\mathcal{C} \backslash \mathcal{S}$. The density in every $T_{i}$ is not less than $\delta^{*}$, so that

$$
\sigma \geq \sigma^{\prime} \geq \delta^{*} \cdot a(U)
$$


Combining (9) and (10), we see that, in fact,

$$
\sigma=\sigma^{\prime}=\delta^{*} \cdot a(U)
$$

This implies that

(A) $\mathcal{S}$ is a solution of the minimum problem;

(B) the union of $\mathcal{S}^{\prime \prime}$ and $\mathcal{C} \backslash \mathcal{S}$ does not contain an unnecessary circle, i.e., $\mathcal{R}=\emptyset$;

(C) all the triangles $T_{1} \ldots, T_{n}$ are light.

Thus, the covering of the hole with admissible circles of minimal total weighted area generates a (possibly different) subdivision of $U$ into light triangles. If there is only one type of light triangles, then the new decomposition of $U$ arises from the original one by rearranging the tiles. This will be the case with the examples discussed in Section 5.

The proof of Theorem 1 is quite straightforward now. Let $\mathcal{C}$ be a covering with circles satisfying condition (i) of Theorem 1 . Let $\mathcal{S}$ be an arbitrary finite subset of $\mathcal{C}$, and let $\mathcal{S}^{\prime}$ be a rearrangement of the circles of $\mathcal{S}$ that together with the rest $\mathcal{C} \backslash \mathcal{S}$ of the covering forms a new covering $\mathcal{C}^{\prime}$. The total weighted areas of $\mathcal{S}$ and $\mathcal{S}^{\prime}$ are the same. Thus, it follows from (A) that $\mathcal{S}^{\prime}$ is also a solution of the extremum problem. This implies that statements (B) and (C) hold for $\mathcal{S}^{\prime}$.

If condition (ii) of Theorem 1 is satisfied as well, then the new subdivision of $U$ into properly joining light triangles leads to the same marked vertices, i.e., the same centers of weighted circles. Therefore, $\mathcal{C}$ and $\mathcal{C}^{\prime}$ are congruent coverings.

This completes the proof of Theorem 1, which applies also to coverings with circles of $n$ different radii and assigned weights.

\section{Applications}

Let $r_{1}=1$ and $r_{2}=r>0$ be radii of admissible circles, and let $w_{1}=1$ and $w_{2}=w>0$ be assigned weights. Consider three admissible circles with radii $r_{i}, r_{j}$, and $r_{k}$ having a point in common, and let $D_{i j k}$ denote the least possible weighted density of these circles in the triangle spanned by their centers. The minimum of the four numbers $D_{111}$, $D_{112}, D_{122}$, and $D_{222}$, i.e., the density in a light triangle, provides a lower bound to the weighted density of covering of the plane by admissible circles. Clearly, $D_{111}=D_{1}$ and $D_{222}=w D_{1}$, where $D_{1}=2 \pi / \sqrt{27}=1.209 \ldots$ For any given $i, j, k$, the triple of circles leading to $D_{i j k}$ is symmetrical and has exactly one point in common, which is a common boundary point (Symmetry Lemma). The shape of the corresponding triangle is uniquely determined, as the density has a single minimum (Quasi-Convexity Lemma).

The weighted density of three circles with radii 1, 1, $r$ is given by formula (6) (Fig. 4), which we write in the form

$$
\delta(x, r, w)=\frac{\pi+2\left(w r^{2}-1\right) \arctan (y /(x+r))}{2 y(x+r)},
$$

where $x$ is the distance of $P$ from $O_{1} O_{2}$, and $y=\sqrt{1-x^{2}}$. We assume that $w r^{2}-1 \neq$ 0 (this will be satisfied in the examples discussed below). The minimum condition 
$f(x, r)=0$, with $f(x, r)$ as in (7), determines $x(r)$ uniquely and reads in our notation

$$
\begin{aligned}
f(x, r, w)= & \frac{(x+r)(1+r x) y}{\left(1-r x-2 x^{2}\right)\left[y^{2}+(x+r)^{2}\right]}+\arctan \frac{y}{x+r} \\
& +\frac{\pi}{2} \frac{1}{w r^{2}-1}=0 .
\end{aligned}
$$

Then

$$
D_{112}=\delta(x(r), r, w) .
$$

An expansion of the 122-configuration by the factor $1 / r$ shows that

$$
D_{122}=w \delta\left(\bar{x}(r), \frac{1}{r}, \frac{1}{w}\right),
$$

where $\bar{x}$ satisfies the equation

$$
f\left(\bar{x}, \frac{1}{r}, \frac{1}{w}\right)=0
$$

obtained from (12) by replacing $x, r$, and $w$ by $\bar{x}, 1 / r$, and $1 / w$.

There exist two promising classes of coverings of the plane, where the Delaunay tiling consists of congruent isosceles triangles. In both cases there are two unit circles at the base and one with radius $r<1$ at the apex. The first class has hexagonal symmetry (Fig. 1) with an angle $\alpha=2 \pi / 3$ at the apex of the tile, while the second has quadratic symmetry (Fig. 2), and the angle at the apex is $\alpha=\pi / 2$. In either case we assign the weight $w=1$ to the unit circles and a weight $w=w(r)>0$ to the circles of radius $r$. The weight $w$ is calculated so that the triangle of smallest density associated with two unit circles and one of radius $r$ has the angle $\alpha=2 \pi / 3$ or $\alpha=\pi / 2$ at the apex. We give the details of the calculation only for the quadratic arrangement, since the procedure for the other arrangement is very similar. In either case, the condition

$$
\tan \frac{\alpha}{2}=\frac{y}{x+r}
$$

has to be satisfied.

Case 1: $\alpha=\pi / 2$. From (16) we obtain

$$
x=\frac{\sqrt{2-r^{2}}-r}{2}=x(r),
$$

where $0<x(r)<1$, for $r<1$. If we choose $w=1 / r^{2}$, then (17) does not satisfy the minimum requirement on the density, i.e., $2 x^{2}+r x-1=0$ (see the proof of the Quasi-Convexity Lemma). Thus we may assume that $w r^{2}-1 \neq 0$, so that the minimum condition is expressed by (12)

$$
f(x(r), r, w)=0,
$$


and this can be transformed into

$$
w r^{2}=\frac{2 \sqrt{2-r^{2}}-r \pi}{2 \sqrt{2-r^{2}}+r \pi} .
$$

In order to satisfy $w>0$, the radius $r$ has to be confined to

$$
0<r<\sqrt{\frac{8}{\pi^{2}+4}}=0.75947 \ldots
$$

Now the weight $w=w(r)$ can be calculated from (19). This equation shows that $w(r) r^{2}$ is a strictly decreasing function. In particular, we observe that

$$
\frac{d w(r)}{d r}<0
$$

By (11), (13), and (17) we get

$$
D_{112}=\frac{\pi}{2} \frac{w r^{2}+1}{1+r \sqrt{2-r^{2}}}
$$

and

$$
\frac{d D_{112}(r)}{d r}<0
$$

The density $D_{122}$ given by (11) and (14) can be written in the form

$$
D_{122}=\frac{\pi w+2\left(1 / r^{2}-w\right) \arctan \left(\sqrt{1-\bar{x}^{2}} /(1 / r+\bar{x})\right)}{2 \sqrt{1-\bar{x}^{2}}(1 / r+\bar{x})} .
$$

We first examine the function $\bar{x}(r)$, i.e., the solution of (15)

$$
F(\bar{x}, r) \equiv f\left(\bar{x}, \frac{1}{r}, \frac{1}{w(r)}\right)=0,
$$

or, explicitly,

$$
\begin{gathered}
\frac{(\bar{x}+1 / r)(1+\bar{x} / r) y}{\left(1-\bar{x} / r-2 \bar{x}^{2}\right)\left[y^{2}+(\bar{x}+1 / r)^{2}\right]}+\arctan \frac{y}{\bar{x}+1 / r} \\
+\frac{\sqrt{2-r^{2}}}{2 r}-\frac{\pi}{4}=0
\end{gathered}
$$

where $y=\sqrt{1-\bar{x}^{2}}$. Straightforward calculation yields

$$
\begin{aligned}
\frac{\partial F}{\partial r}= & \frac{2 y^{3}(\bar{x}+1 / r)}{r^{2}\left(1-\bar{x} / r-2 \bar{x}^{2}\right)^{2}\left(1+2(\bar{x} / r)+1 / r^{2}\right)^{2}} \\
& \times\left[\frac{1}{r}\left(1-\frac{\bar{x}}{r}-2 \bar{x}^{2}\right)-\bar{x}\left(1+2 \frac{\bar{x}}{r}+\frac{1}{r^{2}}\right)\right] \\
& -\frac{1}{r^{2} \sqrt{2-r^{2}}} .
\end{aligned}
$$


Since

$$
\frac{\sqrt{2-r^{2}}}{2 r}-\frac{\pi}{4}>0
$$

for $r$ satisfying (20), (25) implies that $1-\bar{x} / r-2 \bar{x}^{2}<0$, so that, by (26),

$$
\frac{\partial F}{\partial r}<0
$$

while, by (8),

$$
\frac{\partial F}{\partial \bar{x}}>0
$$

Hence

$$
\frac{d \bar{x}(r)}{d r}>0
$$

We shall give an interval of $r$ such that for every point of the interval the density $D_{112}$ is smaller than $D_{111}, D_{122}$, and $D_{222}$.

Many careful calculations based on formulae (19), (22), (24), and (25) suggest that for all $r$ from the interval

$$
I: 0.48770 \leq r \leq 0.50287
$$

the density $D_{112}$ is smaller than the other $D_{i j k}$. A selection of the results is reproduced in the respective columns of Table 1 . Observe that $D_{112}>D_{111}$ for $r_{i}=0.48768$, and $D_{112}>D_{122}$ for $r_{i}=0.50290$. By (21) and (23), the functions $D_{112}(r)$ and $D_{222}(r)=$ $w D_{111}$ are strictly decreasing. An examination of Table 1 shows that indeed $D_{112}<D_{111}$ and $D_{112}<D_{222}$, for $r \in I$. The numerical results make it very likely that $D_{122}(r)$ is also a decreasing function of $r$. However, this appears difficult to prove, as $\bar{x}(r)$ is the root of the rather complicated equation (25). The following proof of the inequality $D_{112}(r)<D_{122}(r)$ for all $r \in I$ avoids this difficulty.

Let $\left[r_{i}, r_{k}\right]$ be any subinterval of $I$, and let $\bar{x}\left(r_{i}\right)=\bar{x}_{i}, \bar{x}\left(r_{k}\right)=\bar{x}_{k}$, and $w\left(r_{k}\right)=w_{k}$. By (21) and (19), the functions $w(r)$ and

$$
\frac{1}{r^{2}}-w(r)=\frac{2 \pi}{2 r \sqrt{2-r^{2}}+r^{2} \pi}
$$

are strictly decreasing, while by (27) the function $\bar{x}(r)$ is strictly increasing. Thus (24) implies the estimate

$$
D_{122}(r) \geq \frac{\pi w_{k}+2\left(1 / r_{k}^{2}-w_{k}\right) \arctan \left(\sqrt{1-\bar{x}_{k}^{2}} /\left(1 / r_{i}+\bar{x}_{k}\right)\right)}{2 \sqrt{1-\bar{x}_{i}^{2}}\left(1 / r_{i}+\bar{x}_{k}\right)} \equiv L\left(r_{i}, r_{k}\right)
$$


Table 1

\begin{tabular}{cccccc}
\hline$r_{i}$ & $D_{111}$ & $D_{112}\left(r_{i}\right)$ & $D_{122}\left(r_{i}\right)$ & $D_{222}\left(r_{i}\right)$ & $L\left(r_{i}, r_{i+1}\right)$ \\
\hline 0.48768 & 1.209200 & 1.209217 & 1.255676 & 1.363456 & 1.256187 \\
0.48770 & 1.209200 & 1.209180 & 1.255575 & 1.363234 & 1.209228 \\
0.49302 & 1.209200 & 1.199279 & 1.228977 & 1.305457 & 1.199322 \\
0.49648 & 1.209200 & 1.192905 & 1.212013 & 1.269106 & 1.192929 \\
0.49873 & 1.209200 & 1.188788 & 1.201119 & 1.245969 & 1.188819 \\
0.50019 & 1.209200 & 1.186127 & 1.194107 & 1.231162 & 1.186139 \\
0.50114 & 1.209200 & 1.184401 & 1.189569 & 1.221614 & 1.184467 \\
0.50175 & 1.209200 & 1.183294 & 1.186664 & 1.215518 & 1.183325 \\
0.50215 & 1.209200 & 1.182569 & 1.184764 & 1.211536 & 1.182596 \\
0.50241 & 1.209200 & 1.182099 & 1.183531 & 1.208954 & 1.182114 \\
0.50258 & 1.209200 & 1.181791 & 1.182725 & 1.207269 & 1.181809 \\
0.50269 & 1.209200 & 1.181592 & 1.182204 & 1.206179 & 1.181621 \\
0.50276 & 1.209200 & 1.181465 & 1.181873 & 1.205486 & 1.181540 \\
0.50280 & 1.209200 & 1.181393 & 1.181683 & 1.205090 & 1.181434 \\
0.50283 & 1.209200 & 1.181339 & 1.181541 & 1.204794 & 1.181375 \\
0.50285 & 1.209200 & 1.181303 & 1.181447 & 1.204596 & 1.181363 \\
0.50286 & 1.209200 & 1.181285 & 1.181399 & 1.204497 & 1.181316 \\
0.50287 & 1.209200 & 1.181267 & 1.181352 & 1.204398 & \\
0.50290 & 1.209200 & 1.181212 & 1.181210 & 1.204102 & \\
$* 0.502385$ & 1.209200 & 1.182143 & 1.183648 & 1.209200 & \\
\hline
\end{tabular}

for all $r \in\left[r_{i}, r_{k}\right]$. Making the assumption that

$$
L\left(r_{i}, r_{k}\right)>D_{112}\left(r_{i}\right)
$$

it follows, by (23), that

$$
D_{122}(r)>D_{112}(r)
$$

for all $r \in\left[r_{i}, r_{k}\right]$. The values $r_{i}$ in Table 1 are selected so that they divide the interval $I$ into a finite number of subintervals $\left[r_{i}, r_{i+1}\right]$, each of which satisfies (30). Thus inequality (31) is proved for every $r \in I$, and $D_{112}$ is smaller than $D_{111}, D_{122}$, and $D_{222}$, as required. Consequently, the Delaunay triangles associated with the covering are light (condition (i) of the theorem). In the considered class of coverings, all Delaunay tiles are congruent right-angled isosceles triangles, and it is easy to show that the marked tilings satisfy condition (ii) of the theorem. Thus, for $r \in I$, each of these coverings is solid.

Case 2: $\alpha=2 \pi / 3$. This case requires only some modifications to the calculations for $\alpha=\pi / 2$. From (16) we obtain

$$
x=\frac{\sqrt{4-3 r^{2}}-3 r}{4}=x(r),
$$

where $0<x(r)<1$, for $r<1 / \sqrt{3}=0.577 \ldots$ Equations (19), (20), and (22) must 
be replaced by

$$
\begin{aligned}
& w r^{2}= \frac{(3 \sqrt{3}-\pi) \sqrt{4-3 r^{2}}-3 r \pi}{(2 \pi+3 \sqrt{3}) \sqrt{4-3 r^{2}}+6 r \pi}, \\
& 0<r<0.40788 \ldots, \\
& D_{112}=\frac{4 \pi}{3 \sqrt{3}} \frac{2 w r^{2}+1}{2-r^{2}+r \sqrt{4-3 r^{2}}} .
\end{aligned}
$$

Inequalities (21) and (23) as well as equality (24) hold unchanged, while in (25) the term $\sqrt{2-r^{2}} / 2 r-\pi / 4$ must be replaced by

$$
\frac{(3 \sqrt{3}-\pi) \sqrt{4-3 r^{2}}-3 r \pi}{18 r+6 \sqrt{4-3 r^{2}}} .
$$

In particular, we remark that inequality (27) continues to hold.

Some results of numerical calculations are collected in Table 2. They suggest that for all $r$ from the interval

$$
I^{\prime}: 0.19194 \leq r \leq 0.25024
$$

the density $D_{112}$ is smaller than $D_{111}, D_{122}$, and $D_{222}$. This can be proved in a similar way as in the case $\alpha=\pi / 2$. Again, values of $r_{i}$ are selected so that (30) is satisfied for every subinterval $\left[r_{i}, r_{i+1}\right]$. All tiles are congruent isosceles triangles with the angle $2 \pi / 3$ at the apex. As before, it is easy to show that the tilings are solid. Therefore, the coverings are also solid for all $r \in I^{\prime}$.

The rows in Tables 1 and 2 marked by an asterisk $(*)$ refer to the weight $w=1$ (i.e., the case where the weighted density and common density coincide). These rows indicate the values $p_{2}$ and $p_{1}$ mentioned in the Introduction. This means that, in a way, our result could be considered an extension of the result in [12].

The ratio of the circumradii of the faces of the Archimedean tiling $(4,8,8)$ is $r=$ $\sqrt{1-(1 / \sqrt{2})}=0.5411 \ldots$, and that of the Archimedean tiling $(3,12,12)$ is $r=$ $\sqrt{(2-\sqrt{3}) / 3}=0.2988 \ldots$ Unfortunately, both ratios are outside the above-mentioned intervals $I$ and $I^{\prime}$, respectively.

\section{Nonsolid Coverings}

Let $\mathcal{C}$ be a covering of the plane by circles with radii $r_{i}(i=1, \ldots, n)$, and let $K(R)$ be a circle with center $O$ and radius $R$. Let $A_{i}(R)$ denote the number of those circles of $\mathcal{C}$ which have radius $r_{i}$ and intersect $K(R)$. In all the cases we consider, the limit

$$
\lim _{R \rightarrow \infty} \frac{A_{i}(R)}{\pi R^{2}}=d_{i} \quad(1 \leq i \leq n)
$$

exists and is called the number-density of the circles of $\mathcal{C}$ having the radius $r_{i}$. The (ordinary) density of $\mathcal{C}$ with respect to the whole plane is

$$
d(\mathcal{C})=\pi \sum_{i=1}^{n} d_{i} r_{i}^{2} .
$$


Table 2

\begin{tabular}{cccccc}
\hline$r_{i}$ & $D_{111}$ & $D_{112}\left(r_{i}\right)$ & $D_{122}\left(r_{i}\right)$ & $D_{222}\left(r_{i}\right)$ & $L\left(r_{i}, r_{i+1}\right)$ \\
\hline 0.19193 & 1.209200 & 1.209212 & 1.489166 & 2.804185 & 1.488955 \\
0.19194 & 1.209200 & 1.209195 & 1.489086 & 2.803751 & 1.209351 \\
0.20694 & 1.209200 & 1.184446 & 1.376972 & 2.230355 & 1.184505 \\
0.21818 & 1.209200 & 1.166550 & 1.301730 & 1.885592 & 1.166607 \\
0.22655 & 1.209200 & 1.153570 & 1.249811 & 1.666135 & 1.153613 \\
0.23276 & 1.209200 & 1.144125 & 1.213300 & 1.520681 & 1.144243 \\
0.23735 & 1.209200 & 1.137244 & 1.187321 & 1.421598 & 1.137371 \\
0.24074 & 1.209200 & 1.132215 & 1.168650 & 1.352633 & 1.132362 \\
0.24324 & 1.209200 & 1.128535 & 1.155150 & 1.303925 & 1.128588 \\
0.24509 & 1.209200 & 1.125827 & 1.145301 & 1.269005 & 1.125928 \\
0.24645 & 1.209200 & 1.123845 & 1.138137 & 1.243922 & 1.123973 \\
0.24745 & 1.209200 & 1.122393 & 1.132909 & 1.225790 & 1.122472 \\
0.24819 & 1.209200 & 1.121320 & 1.129061 & 1.212538 & 1.121468 \\
0.24873 & 1.209200 & 1.120539 & 1.126265 & 1.202956 & 1.120653 \\
0.24913 & 1.209200 & 1.119960 & 1.124200 & 1.195906 & 1.119998 \\
0.24943 & 1.209200 & 1.119527 & 1.122655 & 1.190645 & 1.119577 \\
0.24965 & 1.209200 & 1.119210 & 1.121523 & 1.186801 & 1.119287 \\
0.24981 & 1.209200 & 1.118979 & 1.120702 & 1.184012 & 1.119026 \\
0.24993 & 1.209200 & 1.118806 & 1.120086 & 1.181925 & 1.118829 \\
0.25002 & 1.209200 & 1.118676 & 1.119624 & 1.180363 & 1.118787 \\
0.25008 & 1.209200 & 1.118590 & 1.119317 & 1.179322 & 1.118619 \\
0.25013 & 1.209200 & 1.118518 & 1.119060 & 1.178455 & 1.118642 \\
0.25016 & 1.209200 & 1.118475 & 1.118907 & 1.177935 & 1.118488 \\
0.25019 & 1.209200 & 1.118431 & 1.118753 & 1.177416 & 1.118474 \\
0.25021 & 1.209200 & 1.118403 & 1.118651 & 1.177070 & 1.118511 \\
0.25022 & 1.209200 & 1.118388 & 1.118599 & 1.176897 & 1.118460 \\
0.25023 & 1.209200 & 1.118374 & 1.118548 & 1.176724 & 1.118409 \\
0.25024 & 1.209200 & 1.118359 & 1.118497 & 1.176551 & \\
0.25028 & 1.209200 & 1.118302 & 1.118292 & 1.175859 & \\
$* 0.248378$ & 1.209200 & 1.121048 & 1.128088 & 1.209200 & \\
\hline & & & & & \\
& & & & \\
& & &
\end{tabular}

Let $\mathcal{C}^{\prime}$ be an alternative covering of the plane with circles of the same radii $r_{1}, \ldots, r_{n}$ and number-densities $d_{1}^{\prime}, \ldots, d_{n}^{\prime}$ defined as in (33). Suppose that $\mathcal{C}^{\prime}$ satisfies the two conditions

$$
d_{1}^{\prime}: \cdots: d_{n}^{\prime}=d_{1}: \cdots: d_{n}
$$

and

$$
d\left(\mathcal{C}^{\prime}\right)<d(\mathcal{C})
$$

Let $d_{i}^{\prime} / d_{i}=q(i=1, \ldots, n)$. Then (36) implies

$$
d\left(\mathcal{C}^{\prime}\right)=\pi \sum_{i=1}^{n} d_{i}^{\prime} r_{i}^{2}=\pi q \sum_{i=1}^{n} d_{i} r_{i}^{2}<\pi \sum_{i=1}^{n} d_{i} r_{i}^{2}
$$


whence $q<1$ and

Observe that

$$
d_{i}^{\prime}<d_{i} \quad(i=1, \ldots, n)
$$

$$
d_{i}^{\prime}=\lim _{R \rightarrow \infty} \frac{A_{i}^{\prime}(R)}{\pi R^{2}}=\lim _{R \rightarrow \infty} \frac{A_{i}^{\prime}\left(R+\max 2 r_{k}\right)}{\pi R^{2}} .
$$

From (33), (37), and (38) we deduce that

$$
A_{i}^{\prime}\left(R+\max 2 r_{k}\right)<A_{i}(R)
$$

for sufficiently large $R$ and $i=1, \ldots, n$. Now we remove the set $\mathcal{S}$ of all circles of $\mathcal{C}$ intersecting $K(R)$. Then (39) shows that a proper subset of $\mathcal{S}$ is sufficient for covering the "hole" produced by the removal process. Therefore $\mathcal{C}$ is nonsolid.

A similar statement concerning packings of circles can be proved.

We denote the covering of the plane by circles with radii 1 and $r<1$ in a quadratic and in a hexagonal arrangement by $Q(r)$ and $H(r)$, respectively (see Figs. 2 and 1). In the preceding section these coverings were proved to be solid in the small intervals $I$ and $I^{\prime}$. Although our method works only in $I$ and $I^{\prime}$, there is hardly any doubt that $Q(r)$ and $H(r)$ are solid in larger intervals as well. However, there are intervals of $r$ where $Q(r)$ (or $H(r)$ ) is definitely nonsolid. To show this, it will be sufficient to construct alternative coverings of the plane with the same admissible circles satisfying conditions (35) and (36). For this purpose we consider two different structures.

In the first system the circles of different sizes are separated. One sector of the plane with angle $\alpha$ is covered by unit circles in a regular hexagonal arrangement, the other sector with angle $2 \pi-\alpha$ is covered in a similar way by circles of radius $r$, the density in both sectors being $2 \pi / \sqrt{27}$. The area-densities of these coverings in the respective sectors are

and

$$
\lim _{R \rightarrow \infty} \frac{A_{1}^{\prime}(R) \pi}{\frac{1}{2} R^{2} \alpha}=\frac{2 d_{1}^{\prime} \pi^{2}}{\alpha}
$$

$$
\lim _{R \rightarrow \infty} \frac{A_{2}^{\prime}(R) \pi r^{2}}{\frac{1}{2} R^{2}(2 \pi-\alpha)}=\frac{2 d_{2}^{\prime} \pi^{2} r^{2}}{2 \pi-\alpha},
$$

where $d_{1}^{\prime}$ and $d_{2}^{\prime}$ are the number-densities of the two types of circles. Since the area densities in both sectors are the same, we have

$$
\frac{d_{2}^{\prime}}{d_{1}^{\prime}}=\frac{2 \pi-\alpha}{\alpha} r^{-2} \text {. }
$$

Thus condition (35) is satisfied when

$$
\frac{2 \pi-\alpha}{\alpha}=\frac{d_{2}}{d_{1}} r^{2}
$$

where $d_{1}$ and $d_{2}$ are the number-densities in the original covering. The resulting arrangement forms a covering of the plane with the density

$$
d\left(\mathcal{C}^{\prime}\right)=\frac{2 \pi}{\sqrt{27}} .
$$


The covering $Q(r)$ contains the same number of small and large circles, namely, $d_{2} / d_{1}=$ 1. The fundamental domain is a square with area

$$
t_{4}=\left(r+\sqrt{2-r^{2}}\right)^{2}
$$

and the density of $Q(r)$ is

$$
\delta_{4}(r)=\frac{\left(1+r^{2}\right) \pi}{t_{4}} .
$$

It is easy to show that

$$
\delta_{4}(r)>\frac{2 \pi}{\sqrt{27}},
$$

when $r \leq 0.3$ or $r \geq 0.6030$. Hence $Q(r)$ is nonsolid for such values of $r$.

The covering $H(r)$ contains twice as many small circles as large ones, namely, $d_{2} / d_{1}=2$. The fundamental domain is a rhomb consisting of two equilateral triangles with side-length $\sqrt{3}\left(r+\sqrt{4-3 r^{2}}\right) / 2$ and area

$$
t_{6}=\frac{3 \sqrt{3}\left(r+\sqrt{4-3 r^{2}}\right)^{2}}{8} .
$$

The density of $H(r)$ is

$$
\delta_{6}(r)=\frac{\left(1+2 r^{2}\right) \pi}{t_{6}} .
$$

Again, an easy calculation shows that

$$
\delta_{6}(r)>\frac{2 \pi}{\sqrt{27}}
$$

when $r \geq 0.38$.

Remark 1. The covering $Q(r)$ is nonsolid for $r \leq 0.3$ and for $r \geq 0.6030$. The covering $H(r)$ is nonsolid for $r \geq 0.38$.

We proceed to find another (and, for small values of $r$, more efficient) system to be compared with $Q(r)$. Let one sector with angle $\alpha$ be covered by unit circles in a regular hexagonal arrangement, i.e., with density $2 \pi / \sqrt{27}$. For the other sector (with angle $2 \pi-\alpha$ ), we use a covering of type $H(r)$, i.e., with density $\delta_{6}(r)$. To satisfy condition (35), which requires the ratio of the total number-densities of large and small circles to be $1: 1$, we choose the angle $\alpha$ so that

$$
\frac{2 \pi-\alpha}{\alpha}=\frac{2}{\sqrt{27}} t_{6} .
$$

The density of the combined covering of the plane is

$$
\delta_{06}(r)=\frac{2\left(1+r^{2}\right) \pi}{t_{6}+\sqrt{27} / 2},
$$


where the subscripts 0 and 6 refer to the types of covering in the two sectors. It can be shown that

$$
\delta_{4}(r)>\delta_{06}(r)
$$

for $r \leq 0.34612$.

In a similar way we can construct a combined covering of the plane to be compared with $H(r)$. For one sector we use a covering of type $Q(r)$, i.e., with density $\delta_{4}(r)$, while the complementary sector is covered by circles of radius $r$ in the regular hexagonal arrangement (i.e., with density $2 \pi / \sqrt{27}$ ). Condition (35) requires that the ratio of the total number-densities of large and small circles is $1: 2$. Again, this can be satisfied by an appropriate choice of angles. The density of the combined covering is

$$
\delta_{04}(r)=\frac{\left(1+2 r^{2}\right) \pi}{t_{4}+(\sqrt{27} / 2) r^{2}} .
$$

Comparing this with $\delta_{6}(r)$, we find that

$$
\delta_{6}(r)>\delta_{04}(r)
$$

for $r \geq 0.3591$.

The results are summarized in the following remark.

Remark 2. The covering $Q(r)$ is nonsolid for $0<r \leq 0.3461$ and for $0.6030 \leq r \leq 1$, and $H(r)$ is nonsolid for $0.3591 \leq r \leq 1$.

The results concerning solidity/nonsolidity of $Q(r)$ and $H(r)$ are collected in Theorem 2.

Theorem 2. The covering $Q(r)$ is solid for $0.48770 \leq r \leq 0.50287$, and nonsolid for $0<r \leq 0.3461$ and $0.6030 \leq r \leq 1$. The covering $H(r)$ is solid for $0.19194 \leq r \leq$ 0.25024 and nonsolid for $0.3591 \leq r \leq 1$.

The incircles of the Archimedean tilings $(4,8,8),(3,12,12)$, and $(4,6,12)$ form solid packings (see [14]), but it remains open whether the circumcircles form solid coverings. An argument similar to the one employed above shows that the incircles and circumcircles of the other Archimedean tilings $(3,6,3,6),(3,4,6,4),(3,3,4,3,4)$, $(3,3,3,4,4)$, and $(3,3,3,3,6)$ are nonsolid sets.

\section{Acknowledgments}

We are most grateful to Prof. J. Linhart for his kind assistance in numerical calculations, and to A. C. Florian for preparing the $\mathrm{LT}_{\mathrm{E}} \mathrm{X}$ version of the manuscript. 


\section{References}

1. A. Bezdek, Solid Packing of Circles in the Hyperbolic Plane, Studia Sci. Math. Hungar. 14 (1979), 203-207.

2. G. Blind, Ebene Lagerungen von Kreisen, deren Radien nicht sehr verschieden sind, Ph.D. Dissertation, Technische Hochschule Stuttgart, 1966.

3. G. Fejes Tóth, Covering the Plane with Convex Discs, Acta Math. Acad. Sci. Hungar. 23 (1972), $263-270$.

4. G. Fejes Tóth, Solid Sets of Circles, Studia Sci. Math. Hungar. 9 (1974), 101-109.

5. G. Fejes Tóth, Covering the Plane with Two Kinds of Circles, Discrete Comput. Geom. 13 (1995), 445-457.

6. L. Fejes Tóth, Solid Circle-Packings and Circle-Coverings, Studia Sci. Math. Hungar. 3 (1968), 401-409.

7. L. Fejes Tóth, Lagerungen in der Ebene, aufder Kugel und im Raum, Die Grundlehren der Mathematischen Wissenschaften, Vol. 65, 2nd edn., Springer-Verlag, Berlin, 1972.

8. L. Fejes Tóth, Solid Packing of Circles in the Hyperbolic Plane, Studia Sci. Math. Hungar. 15 (1980), 299-302.

9. L. Fejes Tóth and J. Molnár, Unterdeckung und Überdeckung der Ebene durch Kreise, Math. Nachr. 18 (1958), 235-243.

10. A. Florian, Ausfüllung der Ebene durch Kreise, Rend. Circ. Mat. Palermo (2) 9 (1960), 300-312.

11. A. Florian, Überdeckung der Ebene durch Kreise, Rend. Sem. Mat. Univ. Padova 31 (1961), 77-86.

12. A. Florian, Zum Problem der dünnsten Kreisüberdeckung der Ebene, Acta Math. Acad. Sci. Hungar. 13 (1962), 397-400.

13. B. Grünbaum and G. C. Shephard, Tilings and Patterns, Freeman, San Francisco, CA, 1987.

14. A. Heppes, Solid Circle-Packings in the Euclidean Plane, Discrete Comput. Geom. 7 (1992), $29-43$.

15. A. Heppes and G. Kertész, Packing Circles of Two Different Sizes on the Sphere, in Intuitive Geometry, pp. 357-365, Bolyai Society Mathematical Studies, Vol. 6, János Bolyai Mathematical Society, Budapest, 1997.

16. A. Heppes and J. Molnár, Recent Results in Discrete Geometry, I, Mat. Lapok 9(4) (1960), 330-355 (in Hungarian).

Received August 11, 1998, and in revised form February 22, 1999. 